\title{
Estado emocional en alumnos de Nivel Superior de universidades Públicas/Privadas tras la contingencia del SARS COV2
}

MNC. Alejandra Karina Pérez Jaimes, México.

https://orcid.org/0000-0003-3079-1782

.coord.nutricion@colmexuni.edu.mx

MCS. César Estrada Uziel Estrada Reyes, México.

https://orcid.org/ 0000-0003-4932-2214

cesar.estrada.lic@colmexuni.edu.mx

\section{Campus Licenciaturas}

Grupo Colegio Mexiquense

Dr. César Enrique Estrada Gutiérrez, México.

https://orcid.org/ 0000-0002-0936-8157

cestradag@uaemex.mx

Facultad de Contaduría y Administración

Universidad Autónoma del Estado de México

Recibido: 15 de agosto del 2020

Aceptado: 15 de octubre del 2020

\section{Resumen}

El brote actual del nuevo coronavirus SARS CoV 2 (enfermedad del coronavirus 2019; anteriormente CoV 2019), epicentro en la provincia de Hubei de la República Popular China, se extendido a muchos otros países. En enero de 2020, el Comité de Emergencia de la OMS declaró una emergencia sanitaria mundial basada en el aumento de las tasas de notificación de casos en lugares chinos e internacionales. Teniendo como consecuencia que toda la población a nivel mundial se viera en la necesidad de generar un periodo de confinidad para evitar la propagación del presente virus, en el caso de la educación fue suspendido el modo presencial y en una encrucijada directivos y docentes buscaron transpolar sus clases del aula al modo virtual.Objetivo: asociar el estado emocional en alumnos de Nivel Superior de universidades Públicas/Privadas tras la contingencia del SARS COV2. Metodología: el tipo de investigación analítico, observacional, prospectivo y transversal. Se utilizó una técnica cuantitativa, las variables cuantitativas se utilizaron medidas de tendencia central (media) y de dispersión (rangos mínimos, máximos y DE). El estado emocional y el tipo de universidad fueron evaluados con la prueba estadística de $t$ de student para muestras relacionadas. El Estado Emocional fue medido con la Escala de Rasgo de Metaconocimiento sobre Estados Emocionales (TMMS-24). Resultados: la 
comparación del tipo de universidad con las diferentes subescalas de los Estados Emocionales, de las cuales, en ninguna se encontró una asociación estadísticamente significativa, por lo que el tipo de universidad no condiciona el predominio sobre alguna subescala. Conclusión: La mayoría de los alumnos, tanto de universidades públicas como privadas, tienen un adecuado manejo de su inteligencia emocional, siendo la atención emocional la que se presente con mayor frecuencia como adecuada.

Palabras clave: estado emocional, SARS COV2, nivel superior

\title{
Emotional state in Higher Level students of Public/Private Universities after the SARS COV2 contingency
}

\begin{abstract}
The current outbreak of the new coronavirus SARS CoV 2 (coronavirus disease 2019; formerly CoV 2019), epicentric in the Hubei province of the People's Republic of China, has spread to many other countries. In January 2020, the WHO Emergency Committee declared a global health emergency based on increased case reporting rates in Chinese and international locations. Having as a consequence that the entire population at the world level felt the need to generate a period of confinity to prevent the spread of the virus, In the case of education, the classroom mode was suspended and at a crossroads managers and teachers sought to transfer their classes from the classroom to the virtual mode. Objective: to associate the emotional state in students of Higher Level Methodology: the type of analytical, obasevacional, prospective and transversal research. A quantitative technique was used, the quantitative variables were measures of central trend (mean) and dispersion (minimum, maximum and SD ranges). The emotional state and type of university evaluated with the student $t$ statistical test for related samples. The Emotional State was measured with the Metacognition Trait Scale on Emotional States (TMMS-24). Results: the comparison of the type of university with the different subscales of the Emotional States, of which, in none was found a statistically significant association, so the type of university does not condition the predominance over some subscale. Conclusion: Most students, both from public and private universities, have an adequate management of their emotional intelligence, with emotional attention being the most frequently presented as adequate.
\end{abstract}

Keywords: emotional state, SARS COV2, higher level 


\section{Introducción}

El brote actual del nuevo coronavirus SARS CoV2 (enfermedad del coronavirus 2019; anteriormente CoV19), en la provincia de Hubei de la República Popular China, se extendido a muchos otros países. En enero de 2020, el Comité de Emergencia de la OMS declaró una emergencia sanitaria mundial basada en el aumento de las tasas de notificación de casos en lugares chinos e internacionales. La tasa de detección de casos está cambiando diariamente y se puede seguir en casi tiempo real en el sitio web proporcionado por la Universidad Johns Hopkins y otros foros.

A mediados de febrero de 2020, China soporta la gran carga de morbilidad y mortalidad, mientras que la incidencia en otros países asiáticos, en Europa y América del Norte sigue siendo baja hasta la fecha (Meyer \&Velavan, 2020). Teniendo como consecuencia que toda la población a nivel mundial se viera en la necesidad de generar un periodo de confinidad para evitar la propagación del presente virus, en el caso de la educación fue suspendido el modo presencial y en una encrucijada directivos y docentes buscaron tranpolar sus clases del aula al modo virtual; donde, existieron diferentes limitantes para los alumnos tanto de un desconocimiento de medios digitales, como un disminución emocional ocasionada por la idea de no estar en contacto con sus compañeros e incluso el desarrollo de la clase por el docente.

El poder identificar el estado emocional del alumno durante el presente confinamiento; así como, las diferentes situaciones que presenta al tener educación pública y pagar por la misma, puede generar tensión, frustración y/o depresión en la población estudiantil, disminuyendo su aprovechamiento académico.

\section{Antecendentes}

Estudios como los de De Vries y Navarro (2011) y Suárez (2012) analizan a los egresados universitarios y los comparan según el tipo de institución (pública y privada). Con base en estas investigaciones se sabe que los egresados de las Instituciones de Educación Superior (IES) privadas son más exitosos que los de las públicas en términos de salario, ya que tiene ingresos hasta cuatro veces más altos; respecto a los puestos ocupados, los egresados de IES se colocan como directivos o empleados de oficina mientras que los egresados de escuelas públicas en su mayoría realizan una actividad profesional. En contraste ahora se agrega como situación actual el confinamiento social y el nivel 
emocional, esperando que sin importar la dependencia privada (pública los alumnos no presente ninguna limitante para su desarrollo profesional.

La principal problemática presentada en el confinamiento social ocasionado por la presente contingencia, provoca en el alumnado ansiedad, frustración y depresión tras diferentes factores que conlleva tener clases virtuales y la demanda de trabajo combinado con falta de organización de tiempos presentando una actitud de procrastinación.

Objetivo General: asociar el estado emocional en alumnos de Nivel Superior de universidades Públicas/Privadas tras la contingencia del SARS COV2 Objetivos Específicos: Identificar el estado emocional en alumnos de Nivel Superior y clasificar los alumnos en universidades Públicas/Privadas tras la contingencia del SARS COV2; así como, su edad y género.

\section{Metodología}

La presente investigación será de tipo analítico, observacional, prospectivo y transversal. La población fueron alumnos de Nivel Superior. El tipo de muestreo será no probabilístico a conveniencia, se trabajó con 142 alumnos de Nivel Superior 69 alumnos de la Universidad Autónoma del Estado de México y 73 alumnos de la Universidad Mexiquense de Toluca durante los meses de mayo a junio del 2020.

El Estado Emocional fue medido con la Escala de Rasgo de Metaconocimiento sobre Estados Emocionales (TMMS-24). Para la presente investigación utilizo una técnica cuantitativa, las variables cuantitativas se utilizaron medidas de tendencia central (media) y de dispersión (rangos mínimos, máximos y DE), en cuanto a las variables cualitativas los resultados se generaron con frecuencias y porcentajes. El estado emocional y el tipo de universidad fue evaluado con la prueba estadística de $\mathrm{t}$ de student para muestras relacionadas. Todos los datos se analizaron a través del paquete estadístico SPSS versión 25, tomando como significativo un p valor menor a 0.05.

\section{Resultados}


Tabla 1. Distribución de edad

\begin{tabular}{lcccc}
\hline & Mínimo & Máximo & Media & DE \\
\hline Edad & 18 & 47 & 22.88 & 4.89 \\
\hline
\end{tabular}

\section{Gráfico 1. Distribución de edad.}

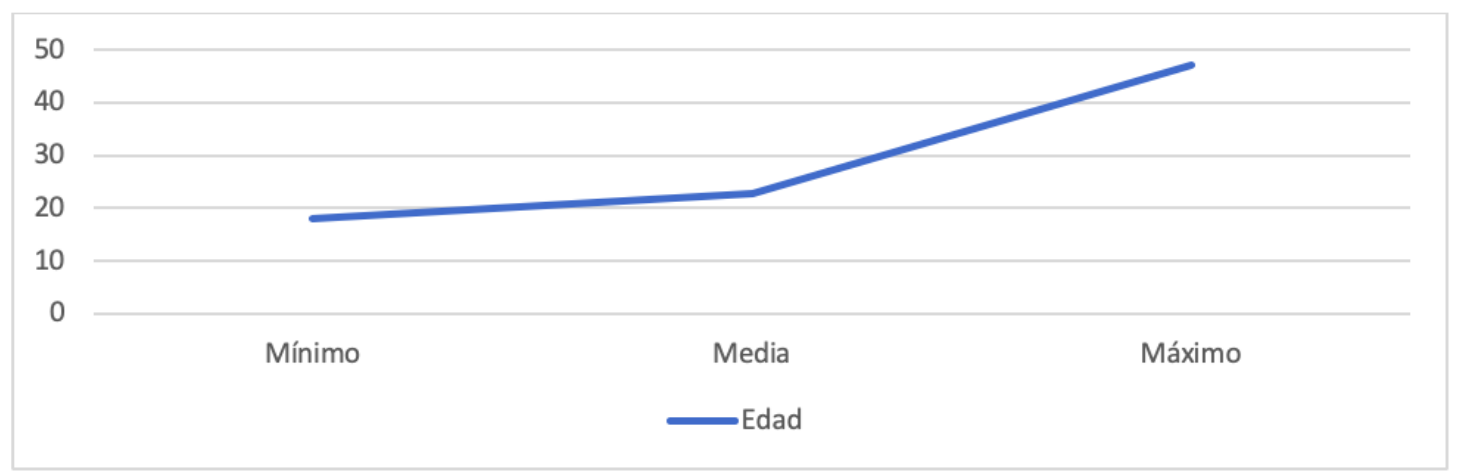

La edad promedio de los alumnos estudiados fue de 22.88 años $( \pm 4.89)$ con un valor máximo de 47 años y mínimo de 18 años.

Tabla 2. Distribución del sexo

\begin{tabular}{lcc}
\hline & Frecuencia & Porcentaje \\
\hline Sexo & 47 & 33.1 \\
Hombre & 95 & 66.9 \\
Mujer & 95 \\
\hline
\end{tabular}

\section{Gráfico 2. Distribución del sexo}

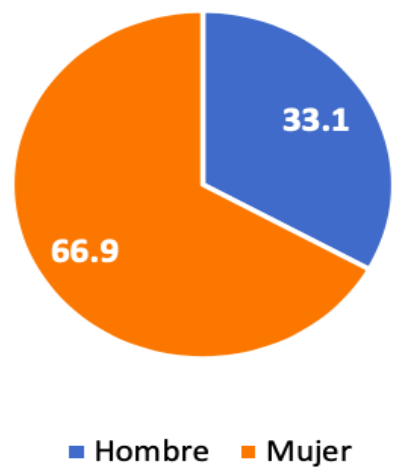

El género predominante fue el femenino con un $66.9 \%(n=95)$, en contraposición al $33.1 \%(\mathrm{n}=47)$ de hombres. 
Tabla 3. Tipo de universidad

\begin{tabular}{lcc}
\hline & Frecuencia & Porcentaje \\
\hline Tipo de universidad & 69 & 48.6 \\
Pública & 73 & 51.4 \\
Privada & & \\
\hline
\end{tabular}

\section{Gráfico 3. Tipo de universidad}

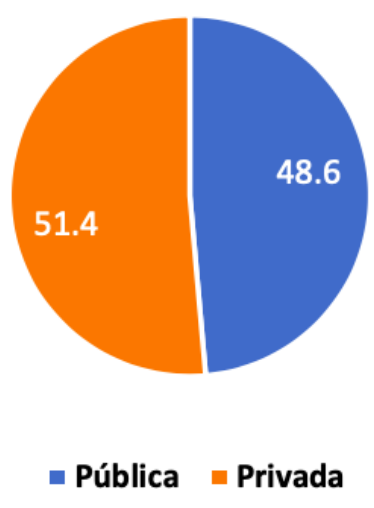

Existió una participación del 51.4\% (n=73) de alumnos de la Universidad Mexiquense de Toluca y un $48.6 \%(n=69)$ de alumnos provenientes de la Universidad Autónoma del Estado de México.

Tabla 4. Comparación de las subescalas de los Estados Emocionales con el sexo

\begin{tabular}{|c|c|c|c|c|c|}
\hline & \multicolumn{2}{|c|}{ Hombre } & \multicolumn{2}{|c|}{ Mujer } & \multirow[t]{2}{*}{$\mathrm{p}^{*}$} \\
\hline & Frecuencia & Porcentaje & Frecuencia & Porcentaje & \\
\hline \multicolumn{6}{|c|}{ Atención emocional } \\
\hline Escasa & 4 & 8.5 & 12 & 12.6 & 0.68 \\
\hline Adecuada & 31 & 66.0 & 63 & 66.3 & 0.68 \\
\hline Excesiva & 12 & 25.5 & 20 & 21.1 & 0.68 \\
\hline $\begin{array}{l}\text { Claridad } \\
\text { sentimien }\end{array}$ & & & & & \\
\hline Escasa & 11 & 23.4 & 17 & 17.9 & 0.20 \\
\hline Adecuada & 23 & 48.9 & 61 & 64.2 & 0.20 \\
\hline Excesiva & 13 & 27.7 & 17 & 17.9 & 0.20 \\
\hline \multicolumn{6}{|c|}{ Reparación emocional } \\
\hline Escasa & 5 & 10.6 & 14 & 14.7 & 0.59 \\
\hline Adecuada & 24 & 51.1 & 52 & 54.7 & 0.59 \\
\hline Excesiva & 18 & 38.3 & 29 & 30.5 & 0.59 \\
\hline
\end{tabular}




\section{Gráfico 4. Comparación de las subescalas de los Estados Emocionales con}

\section{el sexo}

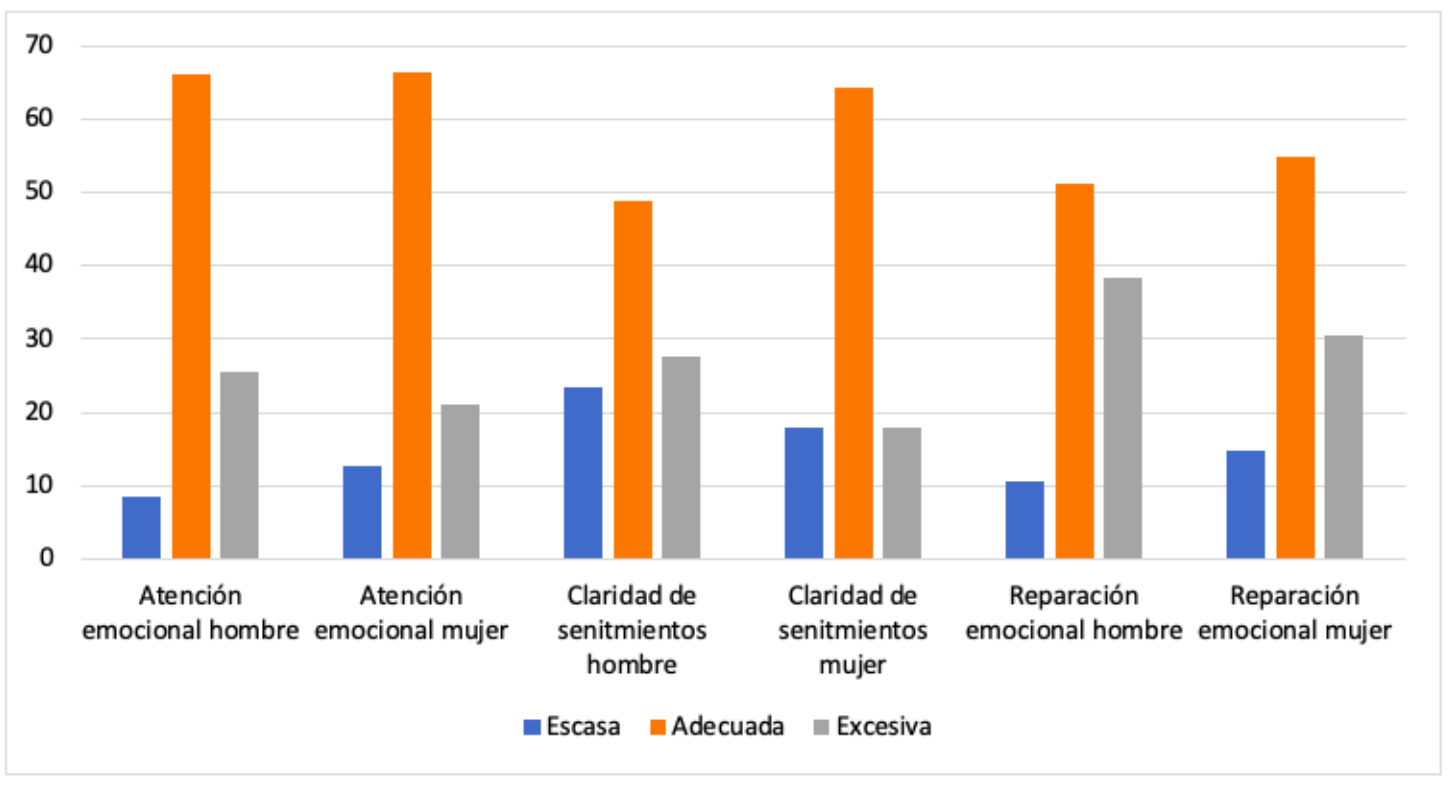

La comparación del sexo con las diferentes subescalas de los Estados Emocionales, de las cuales, en ninguna se encontró una asociación estadísticamente significativa, por lo que el sexo no condiciona el predominio sobre alguna subescala.

Tabla 5. Comparación de las subescalas de los Estados Emocionales con el tipo de universidad

\begin{tabular}{|c|c|c|c|c|c|}
\hline & \multicolumn{2}{|c|}{ Pública } & \multicolumn{2}{|c|}{ Privada } & \multirow[t]{2}{*}{$\mathrm{p}^{*}$} \\
\hline & Frecuencia & Porcentaje & Frecuencia & Porcentaje & \\
\hline \multicolumn{6}{|c|}{ Atención emocional } \\
\hline Escasa & 9 & 13.0 & 7 & 9.6 & 0.24 \\
\hline Adecuada & 41 & 59.4 & 53 & 72.6 & 0.24 \\
\hline Excesiva & 19 & 27.5 & 13 & 17.8 & 0.24 \\
\hline $\begin{array}{l}\text { Claridad } \\
\text { sentimient }\end{array}$ & & & & & \\
\hline Escasa & 14 & 20.3 & 14 & 19.2 & 0.16 \\
\hline Adecuada & 45 & 65.2 & 39 & 53.4 & 0.16 \\
\hline Excesiva & 10 & 14.5 & 20 & 27.4 & 0.16 \\
\hline \multicolumn{6}{|c|}{ Reparación emocional } \\
\hline Escasa & 9 & 13.0 & 10 & 13.7 & 0.51 \\
\hline Adecuada & 34 & 49.3 & 42 & 57.5 & 0.51 \\
\hline Excesiva & 26 & 37.7 & 21 & 28.8 & 0.51 \\
\hline
\end{tabular}




\section{Gráfico 5. Comparación de las subescalas de los Estados Emocionales con el tipo de universidad}

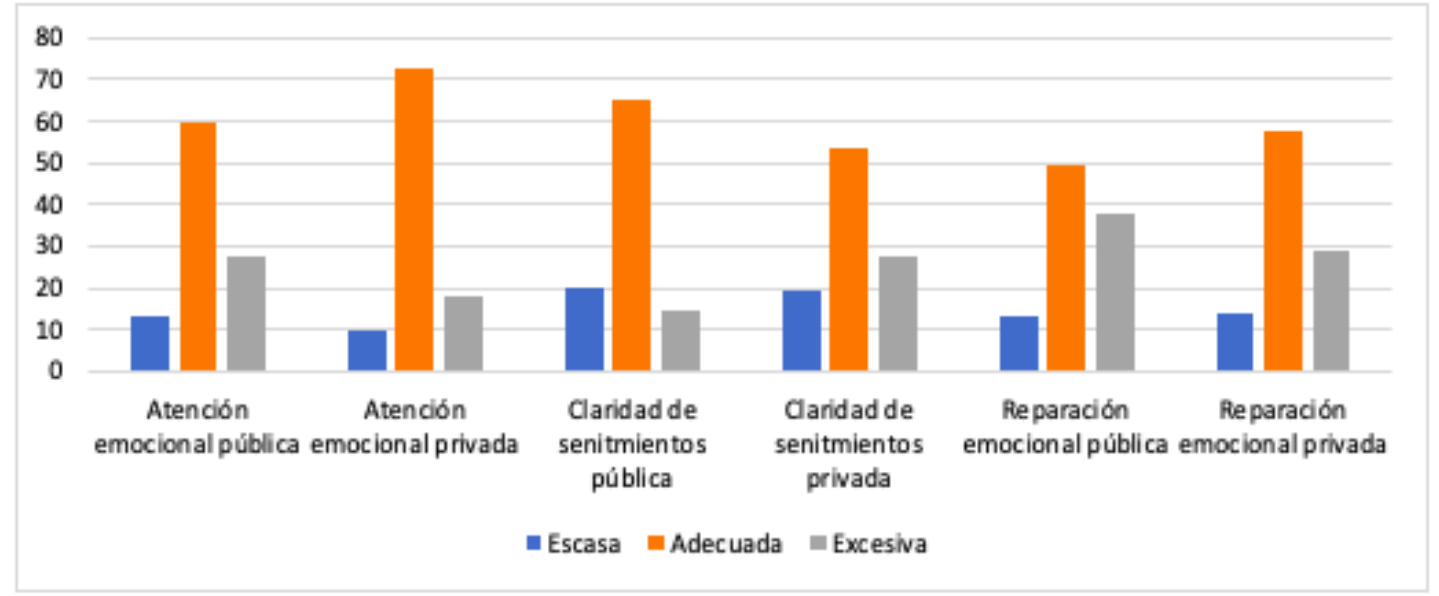

La comparación del tipo de universidad con las diferentes subescalas de los Estados Emocionales, de las cuales, en ninguna se encontró una asociación estadísticamente significativa, por lo que el tipo de universidad no condiciona el predominio sobre alguna subescala.

\section{Discusión de resultados}

La adquisición por parte del estudiante de competencias, habilidades, capacidades y valores favorecen el desarrollo de la Inteligencia Emocional permitiendole reconocer, comprender y regular las propias emociones y la de los demás. Esto implica tres procesos: 1) percibir que sentimos y ser capaces de darle una etiqueta verbal; 2) comprender, integrar lo que sentimos dentro de nuestro pensamiento y saber considerar la complejidad de los cambios emocionales; 3) regular, dirigir y manejar las emociones tanto positivas como negativas de forma eficaz (Fernández-Berrocal y Ramos, 2004).

Las puntuaciones obtenidas por los estudiantes, ubican tanto a los hombres como a las mujeres en un rango que se considera un manejo Adecuado de sus emociones, cabe resaltar que en el Factor Atención que se refiere a la capacidad de sentir y expresar los sentimientos de forma adecuada, el grupo de mujeres obtuvo un mayor porcentaje en la clasificación de escasa, a comparación de los hombre, colocándolas por debajo del rango de Adecuada atención a las emociones; aspecto que llama la atención porque diversos estudios (Sánchez-Núñez, Fernández-Berrocal, Montañez y Latorre, 2008, Fernández- 
Berrocal, 2002, Otero, Martín, León y Vicente, 2009) manifiestan que las mujeres tienden a expresar más fácilmente sus emociones.

Por otra parte, la diferencia encontrada en el Factor Reparación, donde en el grupo de varones fue similar al de las mujeres, no encontrando diferencias estadísticamente significativas; no coincide con estudios que han encontrado diferencias; por ejemplo Sánchez-Núñez, Fernández-Berrocal, Montañez y Latorre, 2008, informaron que no existían diferencias en el tipo de emoción autoinformada por hombres y mujeres, pero sí en la intensidad de éstas, que se refiere este factor que alude a la creencia del sujeto en su capacidad para interrumpir y regular estados emocionales negativos y prolongar los positivos.

Los resultados obtenidos en cuanto al tipo de universidad resultan de especial relevancia, ya que están estrechamente relacionados con el ambiente sociocultural donde se ubica el centro escolar y las características de este. En este sentido y en consonancia con los estudios de Lara, H. (2008), el menciona que, al no contar con las habilidades necesarias para integrarse en su medio, puede compensar las emociones que esto provoca con otro tipo de emociones o acciones.

En esta línea, García Rembao, Valenzuela y Miranda (2012) analizan la competencia socioemocional en una muestra de 78 alumnos de cuatro escuelas situadas en diferentes contextos: urbano, urbano marginal, rural e indígena. Los hallazgos del estudio desvelan, que sí existen diferencias significativas entre los alumnos y su inteligencia socioemocional en los cuatro contextos. A pesar de esto, en la presente investigación, no se encontraron diferencias estadísticamente significativas entre los tipos de universidades, por lo que los estudiantes, tanto de universidades públicas y privadas, tienen un manejo similar de su inteligencia emocional.

\section{Conclusiones}

La mayoría de los alumnos, tanto de universidades públicas como privadas, tienen un adecuado manejo de su inteligencia emocional, siendo la atención emocional la que se presente con mayor frecuencia como adecuada, sin embargo, la claridad de sentimiento es la que presenta los niveles más bajos, por lo que se vuelve importante trabajar en este rubro con los estudiantes. 
No se encontraron asociaciones estadísticamente significativas entre las subescalas de la inteligencia emocional con el tipo de universidad de los estudiantes; sin embargo, puede influir diferentes factores, tiempo, docente, actividades, convivencia en el hogar, limitaciones económicas, etc.

\section{Recomendaciones}

Se sugiere realizar cuestionarios relacionados con estrés, frustración y depresión de los cuales el resultado en ambas poblaciones pueda ser asociados a factores docentes y alumnado que permita apoyarlo durante la presente contingencia, garantizando el rendimiento académico.

\section{Referencias bibliográficas}

De Vries, W., Navarro, Y. (2011). ¿Profesionistas del futuro o futuros taxistas? Los egresados universitarios y el mercado laboral en México. Revista Iberoamericana de Educación Superior, 2(4).

Fernández-Berrocal, P., Ramos, N. (2002). Evaluando la inteligencia emociona. En P. Fernández-Berrocal y N. Ramos (Eds), Corazones Inteligentes. Editorial Kairos: Barcelona.

Fernández-Berrocal, P., Extremera, N., \& Ramos, N. (2004). Validity and reliability of the Spanish modified version of the Trait Meta-Mood Scale. Psychological Reports; 94, 751-755.

García, L., Miranda, J. F., Valenzuela, M. E. (2012). Inteligencia emocional en niños de sexto grado de contextos urbano, urbano marginado, rural e indígena. Revista Electrónica de Investigación Educativa Sonorense; 4, 12, 7-25.

Lara, H. (2008). Investigaciones recientes sobre adolescencia e inteligencia emocional. Red de revistas científicas de América Latina y el Caribe, España y Portugal; $11,2$. 
Otero, C. Martín, E. León, B. y Vicente, F. (2009). Inteligencia emocional en estudiantes de enseñanza secundaria. Diferencias de Género. Revista Galegoportuguesa de Psicoloxía e educación; 17 (1,2), 275-284

Sánchez Núñez, T., Fernández-Berrocal, P., Montañez, J. y Latorre, J.M. (2008). ¿Es la Inteligencia emocional una cuestión de género? Socialización de las competencias emocionales en hombres y mujeres y sus implicaciones. Revista Electrónica de Investigación Psicoeducativa; 15 (6), 455-474.

Velavan, T., Meyer, C.The COVID-19 epidemic. (2020). Tropical Medicine and International Health, 25 (3), 278-280. 